\title{
Ornamental Fishes Recorded from Terai region of West Bengal, India
}

\author{
Debashis Das \\ Department of Zoology, Tufanganj Mahavidyalaya, Coochbehar-736160, WB, India
}

\begin{abstract}
In search of ornamental fishes from the Terai region of West Bengal, an extensive survey was conducted from 2013 to 2016 at the Himalayan foothills from Sonkoshkhola in Alipurduar district to Panitanki in Darjeeling district. Study recorded a total of 128 species of fishes of which 116 are indigenous and 12 are exotic. A total of 58 species of ornamental fishes belonging to 8 orders and 21 families were recorded and documented from the various rivers and lakes of the region. Cypriniformes recorded a highest of 29 species, Siluriformes recorded 12 species and Perciformes recorded 10 species out of the total ornamental fishes recorded from the studied region.
\end{abstract}

Keywords: Terai, fish, ornamental, diversity

\section{Introduction}

The Terai region of West Bengal is the foothills of the Himalaya with dense forest to the North continuous with tea belt and patch of cultivable land to the south. This region is intersected by a number of streams most of which originates from the hills of Bhutan and Sikkim to the north of this region. These rivers swell up enormously during rains and almost dry up in winter. As the tectonic plate of the Brahmaputra river basin tends towards the South-east, therefore most of these rivers enter Bangladesh after flowing their courses in terai region of West Bengal of India. Many marshes, beels, ponds and small pools are present at southern part of Terai region. These wetlands are locally called Dhara, Chhara, Kura, Beels, Doba etc. Luxuriant growths of floating and submerged plants are found in these wetlands along with varieties of planktons and diverse type of fishes.

Many authors published their findings on the ornamental fish recorded from various rivers of North Bengal such as Das (2015) recorded 53 ornamental from Torsa river [1], Dey et al. (2015) reported 58 ornamental from Kaljani river [2], Debnath (2015) recorded 46 ornamental fishes from Gadadhar river [3]. Baro et al. (2014) recorded 49 ornamental fishes from the Sankosh river [4]. Dey and Sarkar (2015) recorded 55 ornamental fishes from Torsa river [5], Dey et al. (2015) reported 46 ornamental fishes from Ghargharia river [6]. Again in a study Sarkar et al. (2015) recorded 24 and 26 ornamental fish species from the river Torsa and Ghargharia respectively [7].

Basu et al. (2012) reported 41 ornamental fish species from Coochbehar district in a report of total ornamental fishes of West Bengal [8]. Paul and Das (2016) recorded 25 potential ornamental fishes from the Coochbehar district of West Bengal [9].

At this juncture the author has conducted an extensive survey to record the ornamental fishes from the terai region of West Bengal in association with the West Bengal Biodiversity Board and UGC (ERO) to document the diversity of fresh water ornamental fishes of sub-Himalayan terai region of West Bengal during 2013 - 2016.

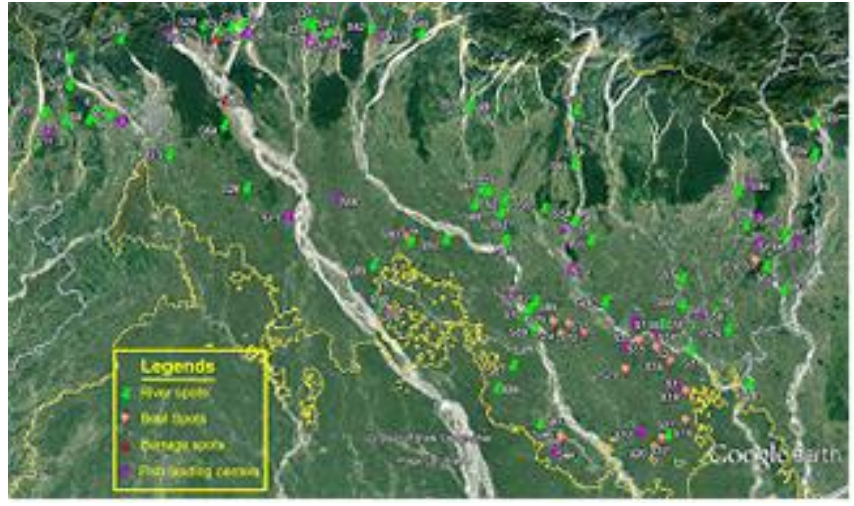

Plate 1: Location of the survey spots in Geo Satellite Map (Courtesy: Google Earth).

\section{Materials and Methods}

The study was conducted at 15 beel spots, 2 barrage spots, 63 river spots (in 50 major rivers flowing through the region) and 24 different fish landing centers throughout the region during a period of $2013-2016$. Location of the survey spots in Google earth map are presented in Plate 1. GPS reading of the location spots were submitted to the ERO-UGC. Local knowledgeable fishermen were interviewed for the selection of the survey spots. Expert fishermen were engaged to capture the fishes from various spots using different types of fishing gears e.g. gill nets, cast nets, dip nets, drag nets etc.

After capturing, a routine documentation was performed using Nikon D7100 DSLR camera, followed by proper preservation using buffered formalin solution following the methods of Bagra and Das (2010) [10]. Collected fishes were identified by their morphometric and meristic characters by consulting the literature of Talwar and Jhingran (1991), Jayaram (1999), Bhattacharyya (2007) and Chanda (2013). Fish Base website was surfed for valid scientific name, and IUCN websites were surfed to evaluate conservation status.

\section{Result and Discussion}

After extensive study it has been noted that 58 fish species have ornamental values out of a total of 128 species recorded 


\section{International Journal of Science and Research (IJSR) ISSN (Online): 2319-7064 \\ Index Copernicus Value (2015): 78.96 | Impact Factor (2015): 6.391}

from the sub-Himalayan terai region of West Bengal of India. A total of 17 species was recorded family Cyprinidae which is highest amongst ornamental fishes recorded from the studied region, out of which 9 belongs to sub-family Barbinae and 7 species from the sub-family Danioninae. Six species of ornamental fishes were recorded from the family
Nemacheilidae under order Cypriniformes and 5 species recorded from the family Sisoridae under order Siluroformes from the sub-Himalayan terai region of West Bengal.

The result is apparently similar with findings of other researchers as noted in the review section of this article.

Table 1: Check list of ornamental fishes recorded from studied spots of Terai region of West Bengal

\begin{tabular}{|c|c|c|}
\hline Scientific name & Common / Local name & $\begin{array}{c}\text { IUCN Status } \\
\text { (ver 3.1:2016) }\end{array}$ \\
\hline \multicolumn{3}{|c|}{ ORDER: OSTEOGLOSSIFORMES: FAMILY: OPHICHTHIDAE } \\
\hline Pisodonophis boro (Hamilton, 1822) & Chei balu & LC \\
\hline \multicolumn{3}{|c|}{ ORDER: CYPRINIFORMES: FAMILY: CYPRINIDAE: SUB FAMILY: Danioninae } \\
\hline Amblypharyngodon mola (Hamilton, 1822) & Maurala & LC \\
\hline Rasbora daniconius (Hamilton, 1822) & Darkina / Dankani / Dadhika & LC \\
\hline Devario devario (Hamilton, 1822) & Chapling / Chebli / Chapchata & LC \\
\hline Devario aequipinnatus (McClelland, 1839) & Chapling & LC \\
\hline Danio dangila (Hamilton, 1822) & Chapling & LC \\
\hline Brachydanio rerio (Hamilton, 1822) & Anju / Zebra fish & NA \\
\hline Bengala elanga (Hamilton, 1822) & Darkina / Elanga & NA \\
\hline \multicolumn{3}{|l|}{ SUB FAMILY: Barbinae } \\
\hline Puntius sophore (Hamilton, 1822) & Deshi Puthi / Jat Puthi & LC \\
\hline Puntius conchonius (Hamilton, 1822) & Kanchan Puthi & LC \\
\hline Puntius gelius (Hamilton, 1822) & Golden barb / Teli Moa / Tepi Mola & LC \\
\hline Puntius terio (Hamilton, 1822) & Teri-puthi & LC \\
\hline Puntius ticto (Hamilton, 1822) & Tita-puthi / Tit-puthi & LC \\
\hline Puntius phutunio (Hamilton, 1822) & Spottedsail barb / Phutuni Puti & LC \\
\hline Systomus sarana (Hamilton, 1822) & Sar-puthi / Saral-puthi & LC \\
\hline Oreichthys crenuchoides (Schafer, 2009) & Pakhna Puti & DD \\
\hline Oreichthys cosuatis (Hamilton, 1822) & Bhuti Puti & LC \\
\hline \multicolumn{3}{|l|}{ SUB FAMILY: Garrinae } \\
\hline Garra mcClellandi (Jerdon, 1849) & Kusma & LC \\
\hline \multicolumn{3}{|l|}{ FAMILY: PSILORHYNCHIDAE } \\
\hline Psilorhynchus sucatio (Hamilton, 1822) & Nou-chata / Balitita / Kakshi & LC \\
\hline Psilorhynchus balitora (Hamilton, 1822) & Baluchata / Titari & LC \\
\hline \multicolumn{3}{|l|}{ FAMILY: NEMACHEILIDAE } \\
\hline Acanthocobitis botia (Hamilton, 1822) & Ghar-poia / Khorkey & LC \\
\hline Nemacheilus corica (Hamilton, 1822) & Kharika & LC \\
\hline Schistura beavani (Gunther, 1868) & Poia & LC \\
\hline Schistura scaturigina (McClelland, 1839) & Poia & LC \\
\hline Schistura multifasciatus (Day, 1878) & Gharpoia & LC \\
\hline Schistura savona (Hamilton, 1822) & Pahari poia & LC \\
\hline \multicolumn{3}{|l|}{ FAMILY: COBITIDAE: SUB FAMILY: Cobitinae } \\
\hline Lepidocephalichthys guntea (Hamilton, 1822) & Poia / Poa & LC \\
\hline Somileptes gongota (Hamilton, 1822$)$ & Guttum /Gongota Loach & NA \\
\hline \multicolumn{3}{|l|}{ SUB FAMILY: Botiinae } \\
\hline Botia dario (Hamilton, 1822) & Botya / Bou Mach / Betrangi & LC \\
\hline Botia lohachata (Chaudhuri, 1912) & Ghutur Poa / Baghlata & NA \\
\hline \multicolumn{3}{|c|}{ ORDER: SILURIFORMES: FAMILY: ERETHISTIDAE } \\
\hline Pseudolaguvia ribeiroi (Hora, 1921) & Batashi / Tinkata & LC \\
\hline Pseudolaguvia shawi (Hora, 1921) & Batashi / Tinkata & LC \\
\hline Erethistoides montana (Hora, 1950) & Bot Magur / Kutakanti / Kurkanti & DD \\
\hline \multicolumn{3}{|l|}{ FAMILY: BAGRIDAE } \\
\hline Batasio tengana (Hamilton, 1822) & Bhutani Tengra & LC \\
\hline \multicolumn{3}{|l|}{ FAMILY: AMBLYCIPITIDAE } \\
\hline Amblyceps mangois (Hamilton, 1822) & Jal-Singi & LC \\
\hline \multicolumn{3}{|l|}{ FAMILY: OLYRIDAE } \\
\hline Olyra longicaudata (McClelland, 1842) & Bot-Singi & LC \\
\hline \multicolumn{3}{|l|}{ FAMILY: SISORIDAE } \\
\hline Nagra assamensis (Sen \& Biswas, 1994) & Ailsa / Ghora kanta & LC \\
\hline Glyptothorax telchitta (Hamilton, 1822) & Telchitta & LC \\
\hline Gogangra viridescens (Hamilton, 1822) & Kaoua Tengra / Kea-Kanta & LC \\
\hline Hara jerdoni (Day, 1870) & Tarkanta / konakanta & LC \\
\hline Conta conta (Hamilton, 1822) & Tiktiki Mach & DD \\
\hline
\end{tabular}

Volume 6 Issue 1, January 2017

www.ijsr.net

Licensed Under Creative Commons Attribution CC BY 


\section{International Journal of Science and Research (IJSR) \\ ISSN (Online): 2319-7064}

Index Copernicus Value (2015): 78.96 | Impact Factor (2015): 6.391

\begin{tabular}{|c|c|c|}
\hline \multicolumn{3}{|l|}{ FAMILY: CHACIDAE } \\
\hline Chaca chaca (Hamilton, 1822) & Chega & NA \\
\hline \multicolumn{3}{|c|}{ ORDER: CYPRINODONTIFORMES: FAMILY: APLOCHEILIDAE } \\
\hline Aplocheilus panchax (Hamilton, 1822) & Te-chokha & LC \\
\hline \multicolumn{3}{|l|}{ ORDER: BELONIFORMES: FAMILY: BELONIDAE } \\
\hline Xenentodon cancila (Hamilton, 1822) & Kankley / Kakley / Khata & LC \\
\hline \multicolumn{3}{|l|}{ FAMILY: SYNGNATHIDAE } \\
\hline Microphis deocata (Hamilton, 1822) & Nol mach / Gharial mach & NT \\
\hline \multicolumn{3}{|c|}{ ORDER: SYNBRANCHIFORMES: FAMILY: MASTACEMBELIDAE } \\
\hline Macrognathus aculeatus (Bloch, 1786) & Guchi / Gota & NA \\
\hline Macrognathus pancalus (Hamilton, 1822) & Pankal / Pakal / Gota & LC \\
\hline \multicolumn{3}{|l|}{ ORDER: PERCIFORMES: FAMILY: CHANNIDAE } \\
\hline Channa orientalis (Bloch \& Schneider, 1801) & Chang & NA \\
\hline \multicolumn{3}{|l|}{ FAMILY: AMBASSIDAE } \\
\hline Chanda nama (Hamilton, 1822) & Nama Chanda & LC \\
\hline Parambassis ranga (Hamilton, 1822) & Ranga Chanda / Lal Chanda & LC \\
\hline Pseudambassis baculis (Hamilton, 1822) & Chanda & LC \\
\hline \multicolumn{3}{|l|}{ FAMILY: NANDIDAE: SUB FAMILY: Nandinae } \\
\hline Nandus nandus (Hamilton, 1822) & Bheda / Meni & LC \\
\hline \multicolumn{3}{|l|}{ SUB FAMILY: Badinae } \\
\hline Badis badis (Hamilton, 1822) & Napit Mach / Bot Koi / Naoa & LC \\
\hline \multicolumn{3}{|l|}{ FAMILY: GOBIIDAE: SUB FAMILY: Gobiinae } \\
\hline Glossogobius giuris (Hamilton, 1822) & Balia / Beley & LC \\
\hline \multicolumn{3}{|c|}{ FAMILY: OSPHRONEMIDAE: SUB FAMILY: Luciocephalinae } \\
\hline Trichogaster fasciatus (Bloch \& Schneider, 1801) & Khalisha / Kholsa & NA \\
\hline Trichogaster labiosa (Day, 1877) & Ranga-kholisha / Kholsa & NA \\
\hline Trichogaster chuna (Hamilton, 1822) & Chuna kholisha (Dhutra) & LC \\
\hline \multicolumn{3}{|c|}{ ORDER: TETRAODONTIFORMES: FAMILY: TETRAODONTIDAE } \\
\hline Tetraodon cutcutia (Hamilton, 1822) & Tepa / Tayapa & LC \\
\hline
\end{tabular}

Note: LC=Least Concern; NT= Near Threatened; EN=Endangered; NA= Not assessed; VU=Vulnerable; DD= Data Deficient.

According to IUCN (Ver 3.1: 2016) Red list 45 recorded species are under the head of 'List Concern', 9 belongs to 'Not Assessed' category, 3 under the head of 'Data Defficient' category and one species belongs to 'Near Threatened'. NT category species recorded from the region is Microphis deocata popularly used as an aquarium fish worldwide. Out of 9, three species under the category of 'NA' Conta conta, Erethistoidae montana and Oreichthys crenuchoides have great importance of aquarium potentiality. They need further study about the abundance status and aquarium survivality status. Authority should promote few species under the category of 'LC' namely Trichogaster chuna, Amblyceps mangois, Badis badis, Aplochelis panchax, Pisodonophis boro etc. for commercial exploration for the economic development of the local people of the subHimalayan terai region of West Bengal.

Plate 2: Ornamental fishes recorded from sub-Himalayan terai region of West Bengal

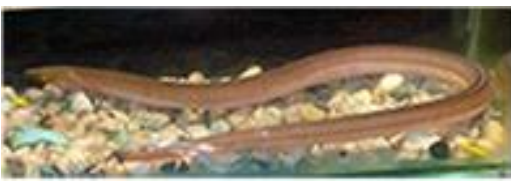

Pisodonophis boro

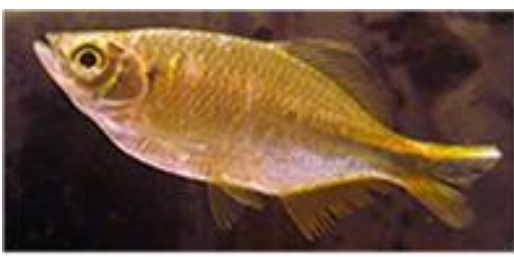

Devario devario

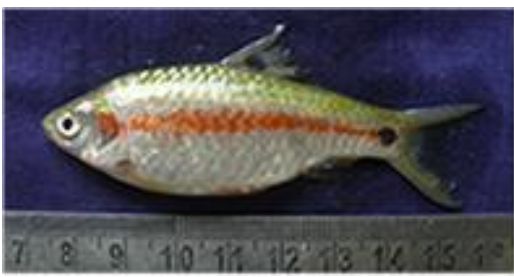

Puntius sophore

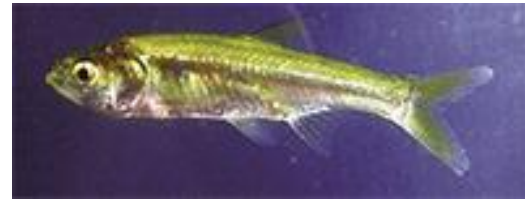

Amblypharyngodon mola

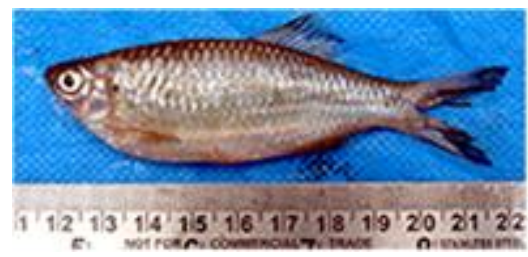

Devario aequipinnatus

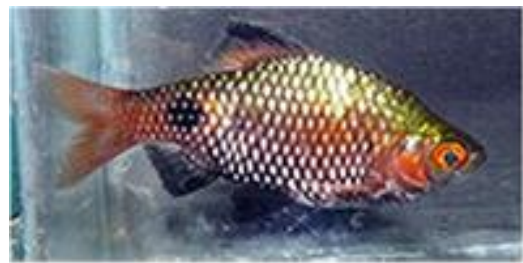

Puntius conchonius

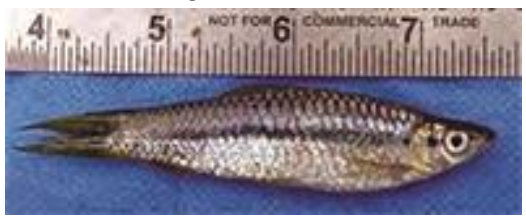

Rasbora daniconius

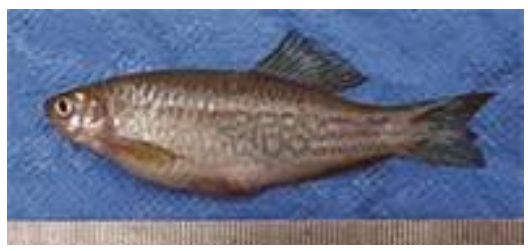

Danio dangila

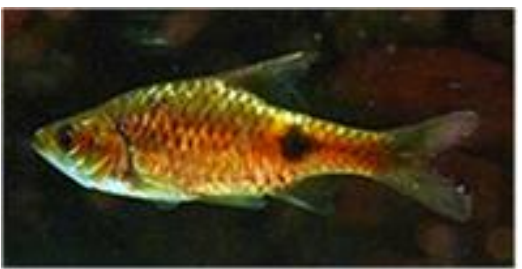

Puntius ticto

Volume 6 Issue 1, January 2017 www.ijsr.net

Licensed Under Creative Commons Attribution CC BY 


\section{International Journal of Science and Research (IJSR) \\ ISSN (Online): 2319-7064}

Index Copernicus Value (2015): 78.96 | Impact Factor (2015): 6.391

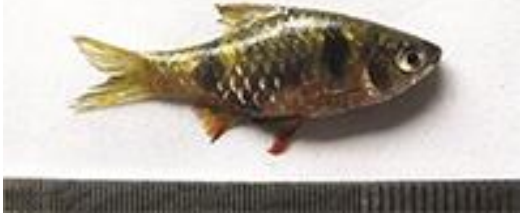

Puntius phutunio
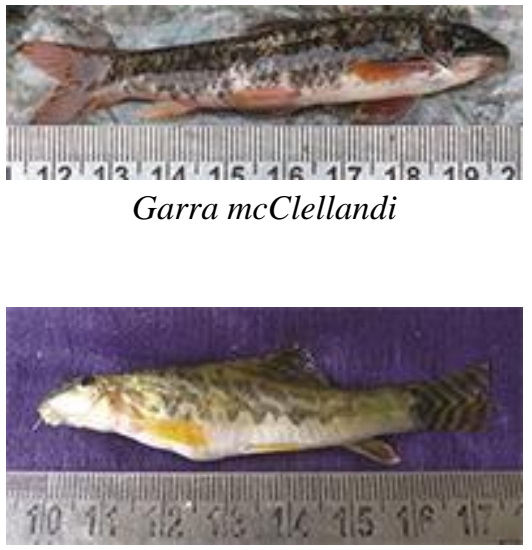

Acanthocobitis botia

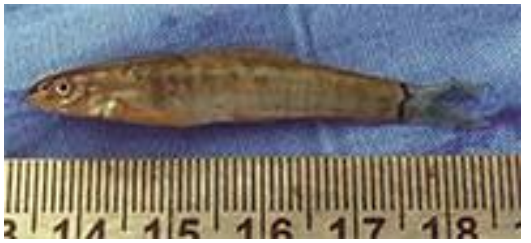

Schistura scaturigina

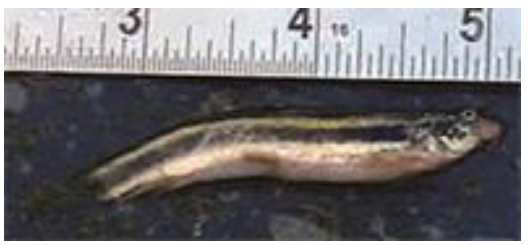

Lepidocephalichthys guntea

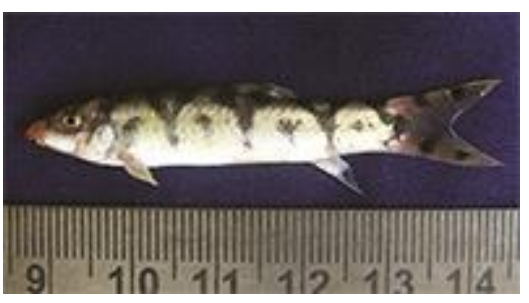

Botia lohachata

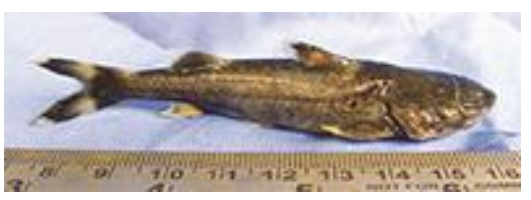

Erethistoides montana

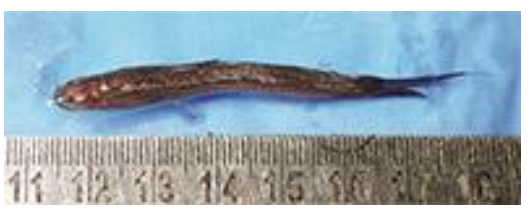

Olyra longicaudata

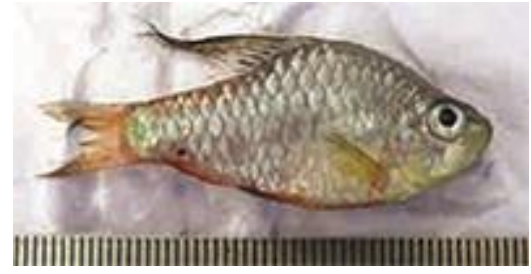

Oreichthys crenuchoides

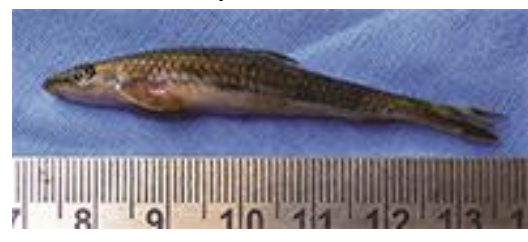

Psilorhynchus sucatio

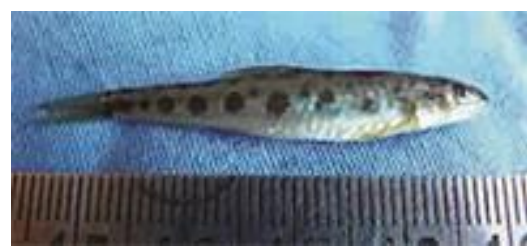

Nemacheilus corica

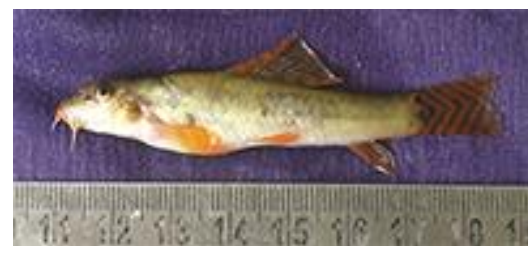

Schistura multifasciatus

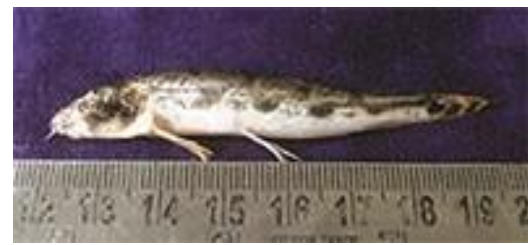

Somileptes gongota

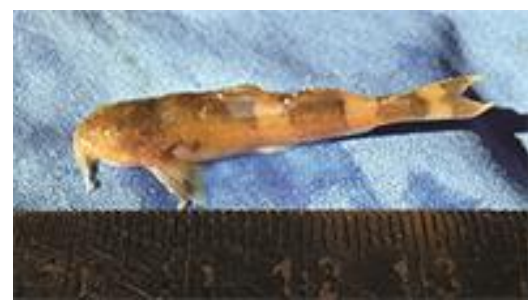

Pseudolaguvia ribeiroi

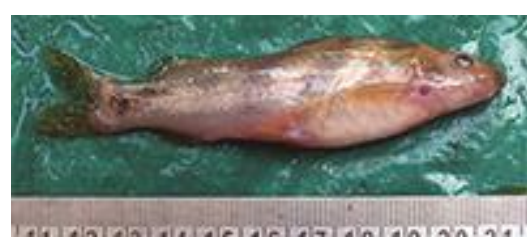

Batasio tengana

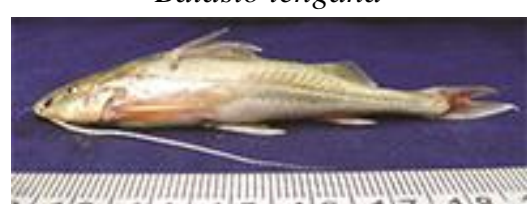

Nagra assamensis

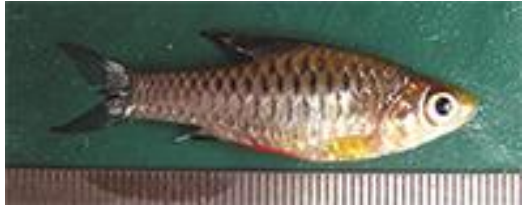

Oreichthys cosuatis

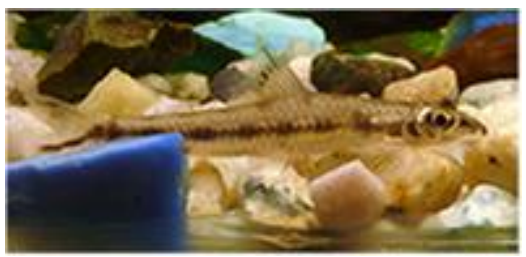

Psilorhynchus balitora

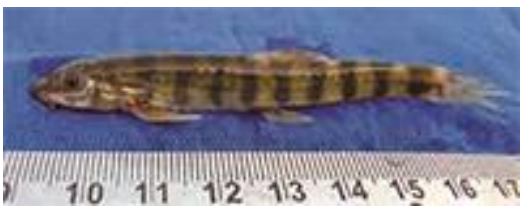

Schistura beavani

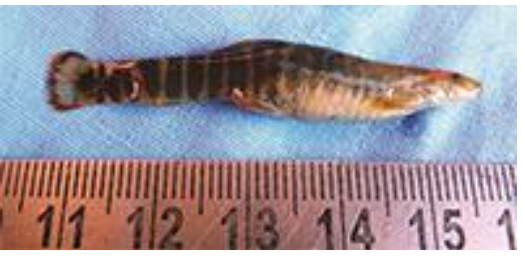

Schistura savona

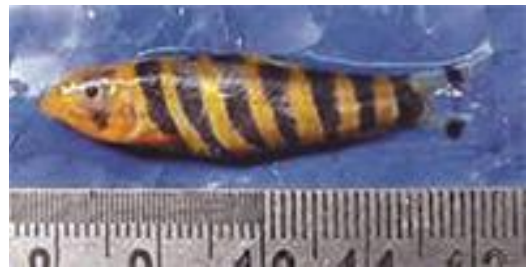

Botia dario

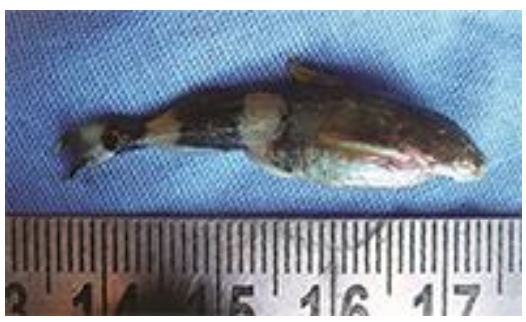

Pseudolaguvia shawi

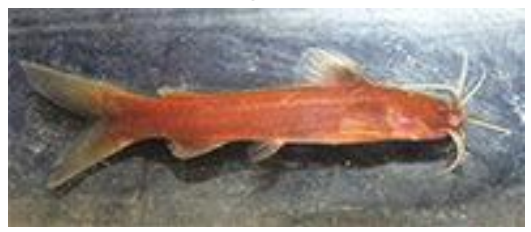

Amblyceps mangois

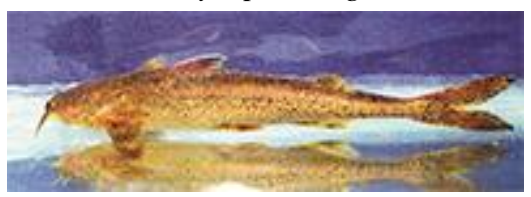

Glyptothorax telchitta

Volume 6 Issue 1, January 2017 www.ijsr.net 


\section{International Journal of Science and Research (IJSR) \\ ISSN (Online): 2319-7064}

Index Copernicus Value (2015): 78.96 | Impact Factor (2015): 6.391

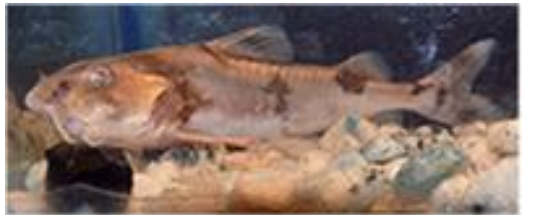

Gogangra viridescens

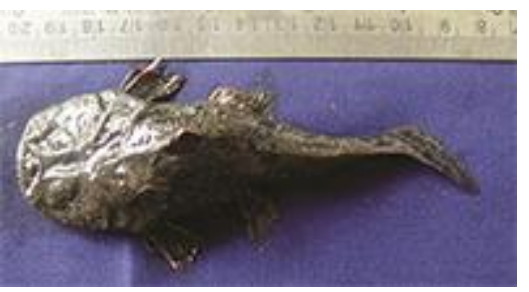

Chaca chaca

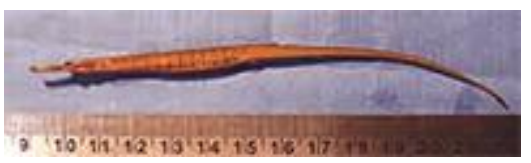

Microphis deocata

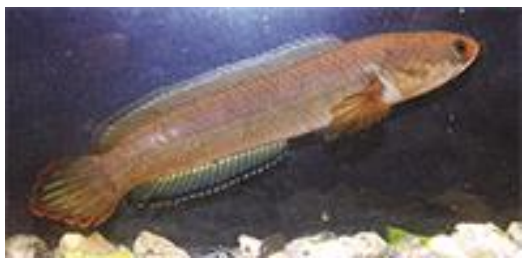

Channa orientalis

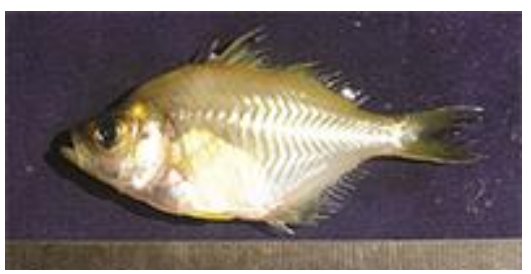

Pseudambassis baculis

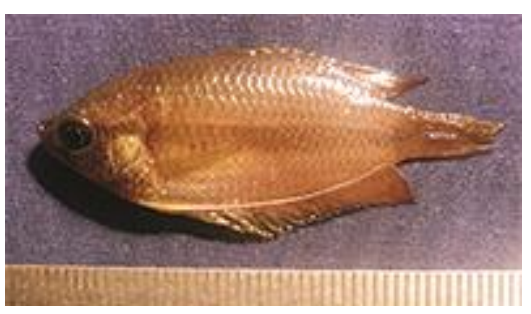

Trichogaster chuna

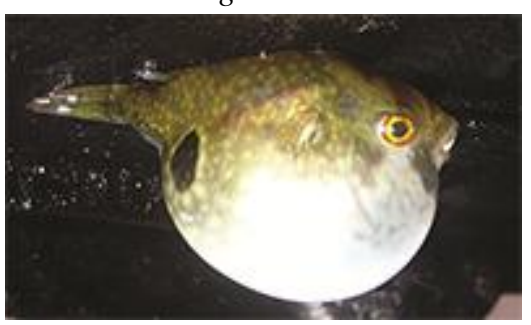

Tetraodon cutcutia

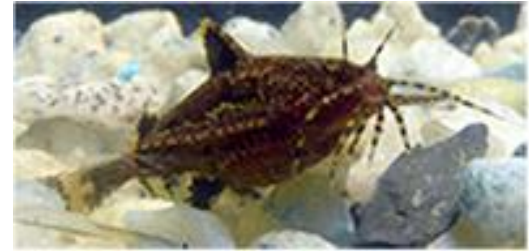

Hara jerdoni

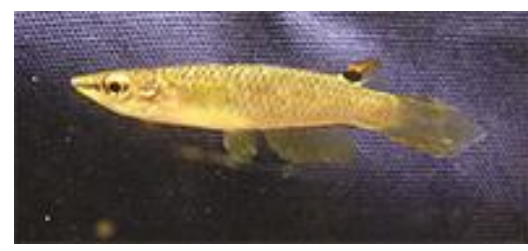

Aplocheilus panchax

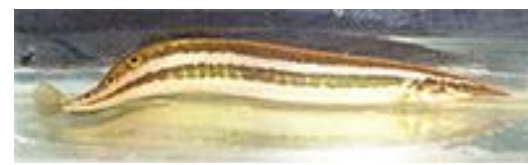

Macrognathus aculeatus

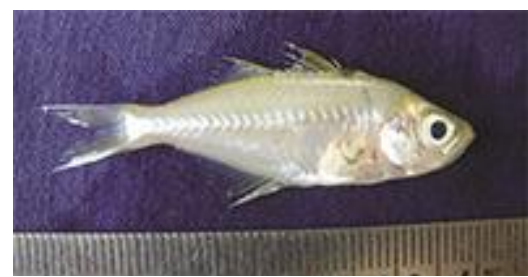

Chanda nama

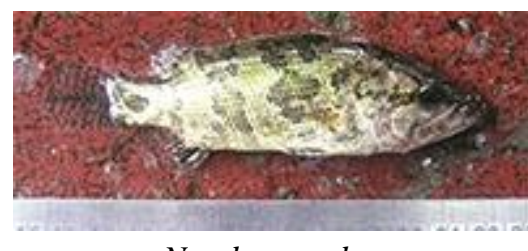

Nandus nandus

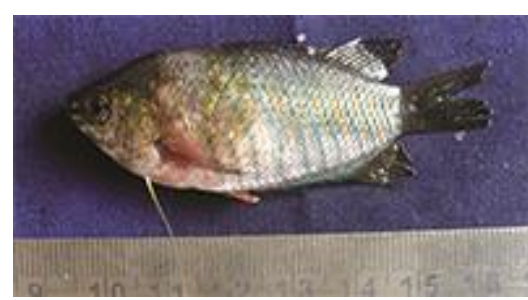

Trichogaster fasciatus

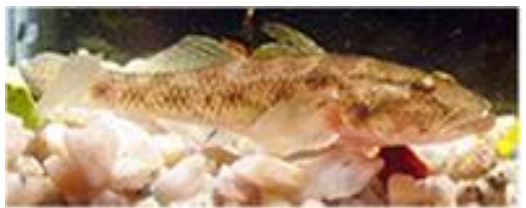

Glossogobius giuris

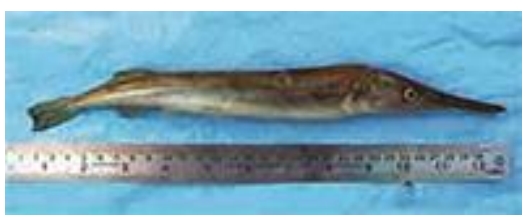

Xenentodon cancila

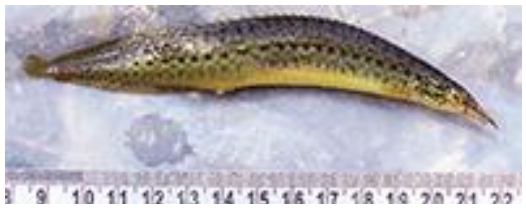

Macrognathus pancalus

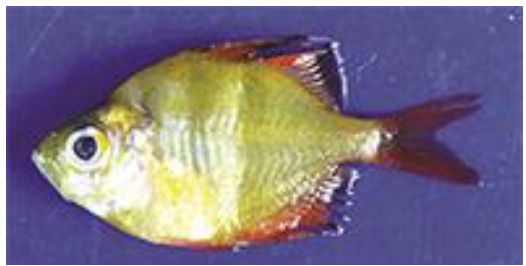

Parambassis ranga

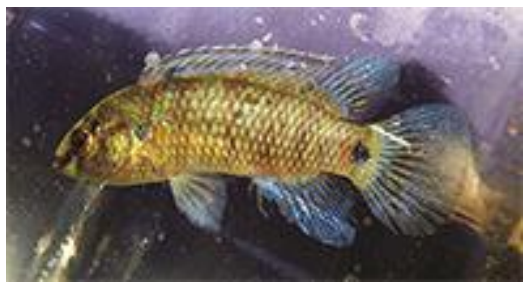

Badis badis

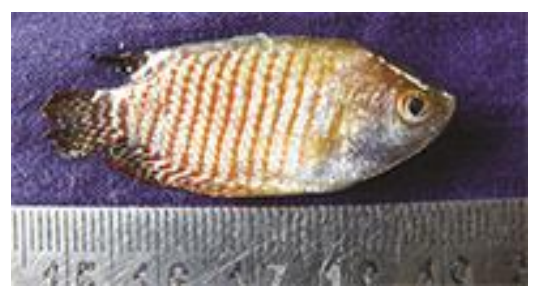

Trichogaster labiosa

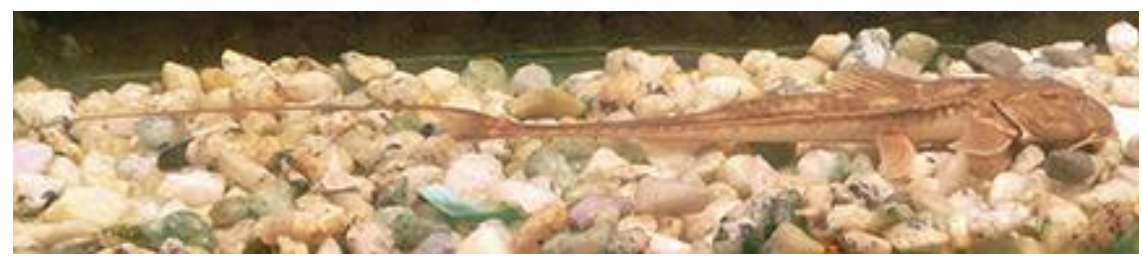

Conta conta

\section{Conclusion}

Out of total 128 fishes recorded from 104 different spots of various wetlands of terai region of West Bengal, 58 species are considered as ornamental fishes. Out of these, one is near threatened according to IUCN. Three species belonging to 'DD' category has very good aquarium value. Few species belong to the category of 'Least concerned' recorded during the study needs proper conservation strategies and commercial exploration for the economic development of the

\section{Volume 6 Issue 1, January 2017 www.ijsr.net}


local people of terai region of West Bengal.

\section{Acknowledgement}

The author is grateful to the West Bengal Biodiversity Board (WBBB) and UGC (ERO) for financial assistance and to the authorities of Tufanganj Mahavidyalaya for giving permission to carry out the work.

\section{References}

[1] Das. D. (2015) Ichthyofaunal diversity of River Torsa and its tributaries at Terai region of West Bengal, India. I.J.S.N., VOL.6 (2) 2015: 256-263.

[2] Dey, A., R. Nur, D. Sarkar, and S. Barat (2015) Ichthyofauna Diversity of River Kaljani in Cooch Behar District of West Bengal, India. Int. J. Pure App. Biosci. 3 (1): 247-256.

[3] S. Debnath (2015) Present Status of Ichthyofaunal Diversity of Gadadhar River at Cooch Behar District, West Bengal, India. Int. J. Pure App. Biosci. 3 (5): 4249.

[4] Baro, D. C., S. Sharma, and R. A. Baishya (2014) Status of ornamental fish diversity of Sonkosh River, Bodoland Territorial Council, Assam, India. Sci Vis 14 (1): 28-33.

[5] Dey, A. and D. Sarkar (2015) In search of Ichthyofauna diversity: A study on Torsa river in Cooch Behar district of West Bengal. Int. J. Pure App. Biosci. 3 (4): 235-241.

[6] Dey, A., A. Mukherjee, D. Sarkar, and N. Ray (2015) Status of indigenous ornamental fish diversity and abundance in Ghargharia river in Coochbehar district of West Bengal. Int. J. Pure App. Biosci. 3 (1): 133-137.

[7] Sarkar, D., A. Dey, and N. Ray (2015) Indigenous Ornamental Fish Diversity in Torsa and Ghargharia: Nature's Conservation Strategy. J. Agric. Technol., 2(1\&2): 21-27.

[8] Basu, A., D. Dutta, and S. Banerjee (2012) Indigenous ornamental fishes of west Bengal. Recent Res. in Sc. \& Tech.. 4(11): 12-21

[9] Paul, A. and Das, D. (2016) Ornamental fishes of Coochbehar District of West Bengal, India. IJSR 5(11): 1827-1832.

[10] Bagra, V. and Das, D. N. (2010) Fish diversity of river Siyom of Arunachal Pradesh, India: A Case Study. Our Nature. 8: 164-169.

[11] Talwar, P. K.,, A. G. Jhingran. (1991) Inland Fishes of India and Adjacent Countries, Vols. 1 and 2. New Delhi, India: Oxford and I.B.H. Publishing Company Ltd.

[12] Jayaram, K. C. (1999) The Freshwater Fishes of Indian Region. Narendra Pub. House. New Delhi

[13] Bhattacharyya, S. (2007) Banglar Maach. Pub. Chairman, West Bengal Biodiversity Board, Kolkata, WB, India

[14]Chanda, B. K. (2013) Uttar Banger Maach: Baichitra O Sanrakshan, Progresive Pub., Kolkata-73. WB, India

[15] www.fishbase.org

[16] www.iucn.org

Volume 6 Issue 1, January 2017

www.ijsr.net

Licensed Under Creative Commons Attribution CC BY 